\title{
Effects of Metformin and Exercise Training, Alone or in Combination, on Cardiac Function in Individuals with Insulin Resistance
}

\author{
Christian Cadeddu - Silvio Nocco (1) - Lucia Cugusi - Martino Deidda • \\ Orru Fabio · Stefano Bandino · Efisio Cossu • Michela Incani • \\ Marco Giorgio Baroni · Giuseppe Mercuro \\ To view enhanced content go to www.cardiologytherapy-open.com \\ Received: October 12, 2015 / Published online: February 1, 2016 \\ (C) The Author(s) 2016. This article is published with open access at Springerlink.com
}

\section{ABSTRACT}

Introduction: In patients affected by insulin resistance (IR), metformin (MET) therapy has been shown to exert its positive effects by improving glucose tolerance and preventing the evolution to diabetes. Recently, it was shown that the addition of metformin to physical training did not improve sensitivity to insulin or peak oxygen consumption (peak VO2). The purpose of this study was to establish the effect of metformin and exercise, separately or in combination, on systolic left ventricular (LV) function in individuals with IR.

Methods: Seventy-five patients with IR were enrolled and subsequently assigned to MET, combination MET and exercise, or exercise

C. Cadeddu and S. Nocco contributed equally to this study.

Electronic supplementary material The online version of this article (doi:10.1007/s40119-016-0057-3) contains supplementary material, which is available to authorized users.

C. Cadeddu · S. Nocco ( $\varangle) \cdot$ L. Cugusi · M. Deidda

O. Fabio $\cdot$ S. Bandino $\cdot$ E. Cossu $\cdot$ M. Incani .

M. G. Baroni · G. Mercuro

Department of Medical Sciences "M Aresu",

University of Cagliari, Strada Statale 554, Km 4.500, 09042 Monserrato, CA, Italy

e-mail: silvionocco@tiscali.it alone. The LV systolic and diastolic functions were evaluated with standard echocardiography tissue Doppler imaging (TDI) and speckle tracking echocardiography at baseline and after 12 weeks of treatment.

Results: MET, administered alone or in association with exercise, improved longitudinal LV function, as evidenced by an increase in systolic (S) wave on TDI, alongside increases in longitudinal global strain and strain rate in comparison to the group undergoing physical training alone. The traditional echocardiographic parameters showed no statistically significant differences among the three groups before or after the different cycles of therapy.

Conclusions: Treatment with MET, either with or without exercise, but not exercise alone, produced a significant increase in global longitudinal LV systolic function at rest. These findings validate the observation that the use of MET alone or in association with exercise has a crucial role to counteract the negative effects of IR on cardiovascular function.

Keywords: Echocardiography; Insulin resistance; Metformin; Physical training; 
Speckle tracking echocardiography; Systolic left ventricular function

\section{INTRODUCTION}

Insulin resistance (IR) is a condition characterized by normal hormone levels producing a sub-optimal biological response, and is considered to be a major etiologic factor in the genesis of non-ischemic heart failure [1]. Therefore, IR leads to a specific myocardial myopathy in which the heart is unable to adequately respond to external stressors by modifying substrate metabolism to increase its energy efficiency. Evidence from several studies determined that IR is crucial in the evolution towards a reduction in cardiovascular performance [2], and is a strong prognostic factor for the subsequent progression to heart failure, independently of all other known cardiovascular risk factors, including diabetes mellitus [3, 4].

The US Diabetes Prevention Program (DPP) showed that changes in lifestyle (i.e., eating a low-fat diet and increasing physical activity) and medical treatment with an insulin-sensitizing drug, such as metformin (MET) reduces the evolution from IR to diabetes mellitus [5]. In a selected population with pre-diabetes, the use of medical treatment with MET has been suggested by several international scientific societies $[6,7]$. However, MET proved to have some collateral negative effects, such as a significant reduction in oxygen consumption, both in healthy individuals [8] and in IR subjects [9].

Exercise training and MET recently proved to increase insulin sensitivity after 3 months of treatment in a population with pre-diabetes. However, the addition of MET appeared to dampen the effect of exercise [10]. In a previous study, we showed a significant increase in peak oxygen consumption (peak VO2) after 12 weeks of exercise alone or in combination with MET, whereas MET alone resulted in no improvement in peak VO2 [11].

Speckle tracking echocardiography (STE) permits the detection of an early subclinical impairment of myocardial function [12]. Using this echocardiographic technique, a reduction in systolic left ventricular (LV) contractile reserve was demonstrated in patients with IR during a physical exercise test [13] or dobutamine stress echocardiography [14].

The aim of the present study was to evaluate a population with IR using STE at baseline and after 3 months of therapy with MET or physical exercise, administered separately or together. The hypothesis of the study was that in IR patients, MET alone or in combination with physical exercise, could prove to exert positive effects on systolic function, as already shown in heart failure patients.

\section{METHODS}

\section{Study Design and Participants}

Male and female subjects were consecutively recruited from individuals screened at the Diabetic Center at our University Hospital (University of Cagliari). No sponsorship was received to perform the study. The study was spontaneous and blinded to the investigators. All of the patients presented with recently identified impaired glucose tolerance (IGT), defined as $2 \mathrm{~h}$ values in the oral glucose tolerance test (OGTT) of 140-199 mg/dL and/ or impaired fasting glucose (IFG), defined as fasting glucose levels of $100-125 \mathrm{mg} / \mathrm{dL}$. OGTT was performed to assess glucose and insulin in the fasting state and every $30 \mathrm{~min}$ up to $2 \mathrm{~h}$ after 
ingesting a glucose load $(75 \mathrm{~g})$. All of the subjects enrolled were affected by IR, defined according to the values of the homeostatic model assessment (HOMA) index (>2.77) described by Bonora et al. [15].

The inclusion criteria for all IR patients were as follows: age 20-55 years, echocardiographic LV ejection fraction (LVEF) $\geq 55 \%$; absence of LV wall motion abnormalities; and normal renal and hepatic function (bilirubin $\leq 1.5 \mathrm{mg}$ / $\mathrm{dL}$, creatinine $\leq 2.0 \mathrm{mg} / \mathrm{dL}$ ). The exclusion criteria were: current smoker; presence of diabetes; hypertension with LV hypertrophy; obesity (overweight patients were accepted with body mass index $<29.9 \mathrm{~kg} / \mathrm{m}^{2}$ ); atrial fibrillation or severe arrhythmias; and moderate-to-severe heart valve disease. Patients with hypertension without LV hypertrophy or dyslipidemia were included in the study.

\section{Study Protocol}

At enrollment, all of the subjects underwent a physical examination, 12-lead electrocardiogram, standard cardiopulmonary (CP) exercise test (CPET), complete blood chemistry, standard echocardiography (M-mode, 2D and Doppler), tissue Doppler imaging (TDI) and evaluation of global longitudinal strain (GLS) and global longitudinal strain rate (GLSR) parameters with STE. All of the patients were subsequently allocated to the three treatment groups (1:1:1) and re-evaluated after 12 weeks: Group M received 12 weeks of MET treatment; Group Ex received 12 weeks of supervised exercise therapy; and Group MEx received 12 weeks of MET therapy plus supervised exercise. Patients receiving therapy with MET started treatment at a dose of $500 \mathrm{mg} /$ day. The dose was increased to the clinical dose of $1000 \mathrm{mg} /$ day in the second week. This dose was administered for the remaining 11 weeks of the study.

\section{Standard and TDI Echocardiography}

Using a commercially available system (Toshiba Artida $^{\mathrm{TM}}$; Toshiba Corp., Tochigi, Japan), echocardiographic images were recorded. LVEF was obtained according to biplane Simpson's rule and considered abnormal at $<55 \%$. From the four-chamber view, a pulsed wave Doppler (PWD) examination of the LV inflow was performed. The sample volume was placed between the mitral leaflet tips to evaluate the early (E) and late (A) diastolic peak velocities. The $\mathrm{E} / \mathrm{A}$ ratio was derived and deceleration time (DecT) of the E wave was measured.

\section{Speckle Tracking Echocardiography (STE)}

A four- and two-chamber view clip was acquired at each evaluation. The longitudinal ventricular function at baseline and after exercise was calculated offline using raw data (Toshiba Corp., Tochigi, Japan). GLS and GLSR were obtained by averaging the strain and strain rate of all LV segments in the four-chamber and two-chamber view.

To achieve homogeneous acquisition of data and images, all of the echocardiographic examinations were performed by the same operator (C.C.). All of the offline measurements were performed by a single investigator (M.D.) who was blinded to the clinical condition of the study participants. The intra-observer variability in our laboratory has been documented previously [16].

\section{Exercise Training Protocol}

The same exercise training protocol was performed in both the exercise groups (Ex and 
MEx). The training program encompassed stretching exercises, and warm-up, central and cool down phases. The central phase included cycle ergometer training at the anaerobic threshold level, which was performed for $60 \mathrm{~min} /$ day four times per week for 12 weeks at our laboratory. After 6 weeks, each subject underwent a CPET to readjust the training workload [17].

\section{Statistical Analysis}

For the anthropometric and clinical characteristics of the three groups, continuous variables were compared using repeated measures analysis of variance (ANOVA). Categorical variables were analyzed with Fisher's exact test. Differences in echocardiographic data were also evaluated using ANOVA. A Bonferroni-corrected two-tailed $P<0.05$ was considered statistically significant.

All procedures followed were in accordance with the ethical standards of the responsible committee on human experimentation (ethics committees of the University of Cagliari, Italy) and with the Helsinki Declaration of 1964, as revised in 2013. Informed consent was obtained from all patients prior to inclusion in the study.

\section{RESULTS}

The study enrolled 75 patients (35 males and 40 females) with a mean [ \pm standard deviation (SD)] age of $46 \quad( \pm 11)$ years. Twenty-five patients were included in each of the three treatment groups. Our population was characterized by a mean $( \pm S D)$ HOMA index of $5.48( \pm 3.8)$, an increased body weight and a reduced mean $( \pm \mathrm{SD})$ peak $\mathrm{VO} 261.83 \pm 12 \%)$ in comparison with theoretical values of a healthy population comparable for age and anthropometric characteristics (Table 1). There were no between-group differences with regard to anthropometric parameters, insulin sensitivity, and cardiovascular risk factors (Table 2). Five subjects from the Ex group and four from the MEx group were excluded from the final analysis due to their inability to regularly carry out the physical training program (training attendance $<70 \%$ ).

Conventional echocardiography revealed normal cardiac chamber size, left ventricular mass and ejection fraction in all patients (Table 3). Diastolic function measured using pulsed Doppler and TDI showed a slight initial diastolic dysfunction when compared with normal values [18]. The systolic function parameters presented as the GLS and GLSR results were consistent with the reference values obtained for healthy populations [19].

The variation in the principal echocardiographic parameters at enrollment and at the 12-week follow-up, and the differences between groups are shown in Table 4. MET alone or in association with exercise training (Groups $\mathrm{M}$ and MEx, respectively) resulted in improvements in the longitudinal left ventricular S wave, GLS and GLSR compared with the Ex Group. Standard echocardiographic parameters did not differ statistically between the groups at baseline or at the 12-week follow-up. Improvements were observed in the S wave, GLS and GLSR in both the $M$ group than the MEx group (Table 5). In the Ex group, none of the examined echocardiographic parameters changed from baseline to post-treatment period (Table 5). However, we observed a trend towards improvement in diastolic function based on a reduction of the end-diastolic filling pressures $\left(\mathrm{E} / \mathrm{E}^{\prime}\right)$ in all three treatment groups (Table 5). 
Table 1 Baseline clinical characteristics of the entire study population $(n=75)$

\begin{tabular}{|c|c|}
\hline Characteristic & Mean $\pm S D^{a}$ \\
\hline Age (years) & $46.2 \pm 11$ \\
\hline Height $(\mathrm{cm})$ & $167.2 \pm 9$ \\
\hline Weight (kg) & $83.3 \pm 11$ \\
\hline BMI $\left(\mathrm{kg} / \mathrm{m}^{2}\right)$ & $29.8 \pm 4.1$ \\
\hline Fasting glucose $(\mathrm{mg} / \mathrm{dL})$ & $112 \pm 14$ \\
\hline Fasting insulin $(\mu \mathrm{U} / \mathrm{mL})$ & $23.7 \pm 14.1$ \\
\hline HOMA index & $5.48 \pm 3.8$ \\
\hline Total cholesterol (mg/dL) & $206 \pm 14$ \\
\hline $\mathrm{HDL}(\mathrm{mg} / \mathrm{dL})$ & $52 \pm 10$ \\
\hline $\mathrm{LDL}(\mathrm{mg} / \mathrm{dL})$ & $131 \pm 24$ \\
\hline Triglycerides (mg/dL) & $146 \pm 55$ \\
\hline Hypertension (\%) & $34.7 \%$ \\
\hline \multicolumn{2}{|l|}{ Glycemic profile (\%) } \\
\hline NG & 17 \\
\hline IFG & 71 \\
\hline IGT & 28 \\
\hline IGT + IFG & 22 \\
\hline Diabetes & 0 \\
\hline \multicolumn{2}{|l|}{ CPET parameters } \\
\hline Peak VO2 (mL/kg/min) & $20.16 \pm 3.72$ \\
\hline$\%$ & $61.83 \pm 12$ \\
\hline $\mathrm{VO} 2(\mathrm{~L} / \mathrm{min})$ & $1.70 \pm 0.41$ \\
\hline$\%$ & $73.6 \pm 12.3$ \\
\hline Work (watts) & $114.6 \pm 31$ \\
\hline $\operatorname{AT}(\mathrm{L} / \mathrm{min})$ & $1.01 \pm 0.25$ \\
\hline AT\% peak VO2 & $48.1 \pm 11$ \\
\hline VO2/work & $9.7 \pm 1.36$ \\
\hline
\end{tabular}

$A T$ anaerobic threshold, $B M I$ body mass index, CPET cardiopulmonary exercise test, $H D L$ high-density lipoprotein, $H O M A$ homeostasis model assessment, IFG impaired fasting glycemia, IGT impaired glucose tolerance, $L D L$ low-density lipoprotein, $N G$ normal glycemic subjects, $S D$ standard deviation, $\mathrm{VO} 2$ oxygen consumption, \% $\mathrm{VO} 2$ in percentage compared to normal values

${ }^{\text {a }}$ Unless otherwise stated

\section{DISCUSSION}

In the present study, we examined the effects of 12 weeks of MET and exercise treatment, separately or in combination, on LV contractile function in a population of patients with IR. In patients with IR, treatment with MET, alone or in association with physical exercise, produced a significant improvement in global longitudinal LV systolic function at rest, as highlighted by speckle tracking and TDI analysis. Also, exercise alone did not improve the above parameters for LV systolic function.

\section{Insulin-resistant Cardiomyopathy}

In humans, most reports in the literature investigating IR and cardiac dysfunction refer to the deterioration of diastolic function [20, 21]. However, IR can lead to impaired myocardial systolic function, potentially through altered calcium homeostasis, increased oxidative stress, altered substrate metabolism, and mitochondrial dysfunction [22]. Thus, there is a link between pre-diabetes and heart failure. A vast majority of individuals with heart failure have stable IR, which, in turn, correlates with the severity of the disease and outcomes [23, 24]. Some years ago Ingelsson et al. showed in a large population that IR is correlated with the incidence of heart failure independently of known risk factors, including diabetes [25].

In the setting of heart failure, the reduced cardiac output and increased adrenergic activation, both of which are responsible for sub-optimal tissue perfusion, could lead to IR and impaired glucose tolerance. Nevertheless, insulin-resistant overt cardiomyopathy has been described in relation to an inefficiency in myocardial energy [26].

IR determines energy inadequacy in cardiomyocytes due to an increased recourse to fatty acids, which are energetically less efficient than glucose. Furthermore, in contrast to those in healthy subjects, the cardiomyocytes of patients with IR are not able to use glucose under conditions of stress. These mechanisms, which are associated with endothelial impairment in IR patients, may 
Table 2 Baseline clinical characteristics of the populations within the study groups

\begin{tabular}{llll}
\hline Characteristic & \multicolumn{2}{l}{ Treatment group } & \\
\cline { 2 - 4 } & $\begin{array}{l}\text { Metformin } \\
\boldsymbol{n}=\mathbf{2 5}\end{array}$ & $\begin{array}{l}\text { Metformin + exercise training } \\
\boldsymbol{n}=\mathbf{2 5}\end{array}$ & $\begin{array}{l}\text { Exercise training } \\
\boldsymbol{n}=\mathbf{2 5}\end{array}$ \\
\hline Age (years) (mean $\pm \mathrm{SD})$ & $47.1 \pm 11$ & $45.5 \pm 12$ & $46.0 \pm 12$ \\
Weight $(\mathrm{kg})($ mean $\pm \mathrm{SD})$ & $84.3 \pm 10$ & $83.5 \pm 13$ & $82.1 \pm 11$ \\
BMI $\left(\mathrm{kg} / \mathrm{m}^{2}\right)($ mean $\pm \mathrm{SD})$ & $28.3 \pm 4.5$ & $30.1 \pm 3.2$ & $31.0 \pm 4.6$ \\
HOMA index $($ mean $\pm \mathrm{SD})$ & $6.07 \pm 4.5$ & $5.71 \pm 3.8$ & $4.67 \pm 4.2$ \\
PeakVO2 $(\mathrm{L} /$ min) & $1.96 \pm 0.38$ & $1.54 \pm 0.50$ & $1.60 \pm 0.38$ \\
Risk factor $n(\%)$ & & & $5(20)$ \\
Smoking & $4(16)$ & $5(20)$ & $12(48)$ \\
Hypertension & $9(36)$ & $8(32)$ & $8(32)$ \\
Hypercholesterolemia & $7(28)$ & $6(24)$ & 0 \\
CAD & 0 & 0 & 0 \\
PAD & 0 & 0 & 5 \\
\hline
\end{tabular}

$B M I$ body mass index, $C A D$ coronary artery disease, $P A D$ peripheral artery disease, $S D$ standard deviation, $V O 2$ oxygen consumption

contribute to the reduction of both cardiopulmonary performance [9] and myocardial contractile reserves [13, 14].

\section{MET and IR}

One treatment option for pre-diabetic patients is the biguanide MET. In diabetic patients, MET has been associated with improved outcomes [27]. In the US DPP, MET was demonstrated to reduce the evolution from a condition of glucose intolerance to diabetes [28, 29]. A beneficial effect on systolic function after 3 months of MET therapy has been previously described in animal models of heart failure, in which it was demonstrated that administration of the drug provides significant cardio-protective effects and improves LV function [30, 31]. In patients with heart failure, the use of MET was shown to cause an increase in systolic function with better outcomes compared to other treatments [32, 33]. Wong et al. recently demonstrated a positive effect of MET treatment on LV function in IR patients with advanced heart failure [34]. They showed that LVEF recovers only when the treatment with MET is accompanied by a decrease in brain natriuretic peptide, thereby suggesting that MET may improve systolic function by decreasing cardiac overload. The present study, using the reliable STE technique, confirmed the ability of MET to significantly improve LV systolic function in an IR population.

The detailed mechanisms by which MET exerts its beneficial effects appear to be related to a decrease in free fatty acid levels, reduction of myocardial lipid accumulation, diminished perivascular fibrosis, and AMPK-eNOS signal activation [30]. However, MET has several limitations concerning its use, and various adverse events have been reported. MET may 
Table 3 Echocardiographic data for the entire study population $(n=75)$

\begin{tabular}{lc}
\hline Parameter & Mean \pm SD \\
\hline EDD $(\mathrm{mm})$ & $47.45 \pm 4.34$ \\
EDV $(\mathrm{mL})$ & $80.62 \pm 20.32$ \\
Left ventricular mass $(\mathrm{g})$ & $145.47 \pm 5.68$ \\
EF $(\%)$ & $66.67 \pm 4.99$ \\
LAA $\left(\mathrm{cm}^{2}\right)$ & $18.56 \pm 3.45$ \\
DcT $(\mathrm{s})$ & $0.195 \pm 0.05$ \\
IVRT $(\mathrm{s})$ & $0.11 \pm 0.06$ \\
E/E' & $9.51 \pm 3.65$ \\
S wave $(\mathrm{cm} / \mathrm{s})$ & $7.11 \pm 1.13$ \\
GLS $(\%)$ & $24.6 \pm 10.8$ \\
\hline
\end{tabular}

$D c T \mathrm{E}$ wave deceleration time, $E D D$ end-diastolic diameter, $E D V$ end-diastolic volume, $E / E^{\prime} \mathrm{E}$ wave peak velocity/ $\mathrm{E}^{\prime}$ wave peak velocity ratio, $E F$ left ventricular ejection fraction, GLS global longitudinal strain, GLSR global longitudinal strain rate, IVRT iso-volumic relaxation time, $L A A$ left atrium area, $S D$ standard deviation

cause a decrease in VO2 in subjects with no IR [8], and our previous data have demonstrated the same effect in patients with IR [9]. It is of importance that this effect does not occur in every patient but mainly in those with a lower degree of IR; in contrast, CP performance is significantly increased in subjects with a high degree of IR. In patients with heart failure, MET significantly improves IR and the pulmonary ventilation/ventilator carbon dioxide slope, leading to significant weight loss; however, it is unable to increase the peak VO2 [34].

\section{The Association Between Physical Training and MET in IR}

Regular exercise increases the peripheral sensitivity to insulin mainly of skeletal muscle, largely by stimulating AMPK $[35,36]$. The American Diabetes Association strongly recommends exercise as a cornerstone therapy for the prevention of diabetes, and the combination of exercise and MET has been proposed in place of each of the two separate therapies [17]. Currently available data suggest an advantage of the addition of metformin to physical training in terms of weight loss [37] but negligible or no effect on IR [38]. Insulin sensitivity in pre-diabetic subjects can be increased by physical exercise, but the association with MET does not determine a further advantage in terms of sensitivity to the hormone and can even attenuate the effect of the physical training [10].

In a previous study, we showed that the association with physical training could compensate the negative effects of MET on cardiopulmonary performance. Furthermore, we demonstrated that physical exercise, when personalized and supervised, affected cardiopulmonary parameters and quality of life similar to the combination of MET and exercise [11]. Moreover, there are limited data for the effect of physical training alone or in combination with MET on LV cardiac function. Stewart et al. showed that 6 months of exercise had no effect on cardiac dimensions or on LV diastolic function despite a significant increase in cardiopulmonary performance in terms of peak VO2 [39].

To our knowledge, the present results show for the first time that physical training alone does affect LV cardiac function despite the improvements in cardiopulmonary performance. However, the association between supervised exercise and MET does not reduce the positive effects of the drug on the indices of LV systolic function and retains its positive influence on exercise capacity in terms of peak VO2. At present, however, it remains unknown whether the beneficial effects on the indices of myocardial performance at rest can be 
Table 4 Differences in echocardiographic parameters between pre- and post-treatment

\begin{tabular}{|c|c|c|c|}
\hline \multirow[t]{2}{*}{ Parameter } & \multicolumn{3}{|c|}{ Treatment group (mean $\pm S D)$} \\
\hline & $\begin{array}{l}\text { Metformin } \\
n=25\end{array}$ & $\begin{array}{l}\text { Metformin }+ \text { exercise training } \\
n=21\end{array}$ & $\begin{array}{l}\text { Exercise training } \\
n=20\end{array}$ \\
\hline $\operatorname{EDD}(\mathrm{mm})$ & $-1.78 \pm 1.21$ & $1.60 \pm 1.49$ & $0.82 \pm 1.22$ \\
\hline IVS (mm) & $0.46 \pm 15.14$ & $-1.68 \pm 16.79$ & $0.37 \pm 16.98$ \\
\hline Left ventricular mass $(\mathrm{g})$ & $-4.69 \pm 28.00$ & $-5.4 \pm 29.21$ & $-6.06 \pm 16.99$ \\
\hline $\mathrm{EDV}(\mathrm{mL})$ & $-4.68 \pm 15.15$ & $1.45 \pm 9.86$ & $3.49 \pm 11.54$ \\
\hline $\mathrm{EF}(\%)$ & $0.68 \pm 3.61$ & $-4.23 \pm 5.16$ & $-1.88 \pm 4.12$ \\
\hline $\operatorname{IVRT}(s)$ & $0.00 \pm 0.04$ & $-0.01 \pm 0.04$ & $0.00 \pm 0.06$ \\
\hline $\mathrm{E} / \mathrm{E}^{\prime}$ & $-0.65 \pm 1.25$ & $-0.57 \pm 1.27$ & $-0.45 \pm 1.32$ \\
\hline$S$ wave $(\mathrm{cm} / \mathrm{s})$ & $0.82 \pm 4.65^{\mathrm{a}}$ & $1.07 \pm 3.84^{\mathrm{a}}$ & $-0.08 \pm 3.39$ \\
\hline GLS (\%) & $9 \pm 5^{\mathrm{a}}$ & $15 \pm 7^{\mathrm{a}}$ & $-2 \pm 4.7$ \\
\hline $\operatorname{GLSR}\left(s^{-1}\right)$ & $0.53 \pm 0.41^{\mathrm{a}}$ & $0.55 \pm 0.59^{\mathrm{a}}$ & $0.05 \pm 0.39$ \\
\hline
\end{tabular}

$E D D$ end-diastolic diameter, $E D V$ end-diastolic volume, $E / E^{\prime} \mathrm{E}$ wave peak velocity/ $\mathrm{E}^{\prime}$ wave peak velocity ratio, $E F$ left ventricular ejection fraction, $E x$ exercise training group, GLS global longitudinal strain, GLSR global longitudinal strain rate, $I V R T$ iso-volumic relaxation time, IVS inter-ventricular septum, $S D$ standard deviation

a $P<0.05$ vs Ex

Table 5 Echocardiographic parameter data pre- and post-treatment

\begin{tabular}{|c|c|c|c|c|c|c|}
\hline \multirow[t]{3}{*}{ Parameter } & \multicolumn{6}{|c|}{ Treatment group, mean \pm SD } \\
\hline & \multicolumn{2}{|c|}{ Metformin $n=25$} & \multicolumn{2}{|c|}{$\begin{array}{l}\text { Metformin }+ \text { exercise training } \\
n=21\end{array}$} & \multicolumn{2}{|c|}{ Exercise training $n=20$} \\
\hline & $\overline{\text { Pre }}$ & Post & $\overline{\text { Pre }}$ & Post & $\overline{\text { Pre }}$ & Post \\
\hline $\operatorname{EDD}(\mathrm{m})$ & $47.34 \pm 3.33$ & $45.56 \pm 5.55$ & $46.90 \pm 5.94$ & $48.50 \pm 4.71$ & $47.56 \pm 5.30$ & $48.38 \pm 4.98$ \\
\hline IVS (mm) & $8.61 \pm 0.97$ & $9.07 \pm 1.51$ & $10.13 \pm 2.09$ & $8.45 \pm 1.50$ & $7.95 \pm 1.20$ & $8.32 \pm 0.778$ \\
\hline MASS (g) & $145.82 \pm 31.80$ & $141.13 \pm 47.69$ & $144.40 \pm 35.15$ & $139.00 \pm 24.45$ & $147.56 \pm 15.01$ & $141.5 \pm 23.44$ \\
\hline $\mathrm{EDV}(\mathrm{mL})$ & $71.66 \pm 14.55$ & $66.98 \pm 11.45$ & $78.41 \pm 21.13$ & $79.86 \pm 14.11$ & $75.40 \pm 15.20$ & $78.89 \pm 13.00$ \\
\hline $\mathrm{EF}(\%)$ & $66.77 \pm 4.60$ & $67.45 \pm 6.72$ & $68.73 \pm 8.94$ & $64.50 \pm 7.46$ & $67.54 \pm 8.34$ & $65.66 \pm 7.34$ \\
\hline $\operatorname{IVRT}(s)$ & $0.07 \pm 0.02$ & $0.07 \pm 0.02$ & $0.09 \pm 0.02$ & $0.08 \pm 0.02$ & $0.08 \pm 0.02$ & $0.08 \pm 0.02$ \\
\hline $\mathrm{E} / \mathrm{E}^{\prime}$ & $8.65 \pm 2.89$ & $9.30 \pm 2.23$ & $9.00 \pm 0.73$ & $8.43 \pm 1.32$ & $8.26 \pm 0.66$ & $7.81 \pm 0.97$ \\
\hline$S$ wave $(\mathrm{cm} / \mathrm{s})$ & $6.95 \pm 1.24$ & $7.77 \pm 0.87^{\mathrm{a}}$ & $6.96 \pm 1.34$ & $7.97 \pm 0.94^{\mathrm{a}}$ & $7.43 \pm 1.56$ & $7.35 \pm 0.91$ \\
\hline GLS (\%) & $27 \pm 12$ & $36 \pm 13^{\mathrm{a}}$ & $22 \pm 10$ & $37 \pm 11^{\mathrm{a}}$ & $24 \pm 11$ & $22 \pm 10$ \\
\hline GLSR $s^{-1}$ & $1.10 \pm 0.50$ & $1.63 \pm 0.57^{\mathrm{a}}$ & $1.31 \pm 0.59$ & $1.86 \pm 0.6^{\mathrm{a}}$ & $0.83 \pm 0.14$ & $0.88 \pm 0.12$ \\
\hline
\end{tabular}

$E D D$ end-diastolic diameter, $E D V$ end-diastolic volume, $E / E^{\prime} \mathrm{E}$ wave peak velocity/ $\mathrm{E}^{\prime}$ wave peak velocity ratio, $E F$ left ventricular ejection fraction, Ex exercise training group, GLS global longitudinal strain, GLSR global longitudinal strain rate, $I V R T$ iso-volumic relaxation time, IVS inter-ventricular septum, MASS left ventricular mass, $S D$ standard deviation

a $P<0.05$ vs Pre-treatment 
preserved during physical activity in terms of contractile reserve and cardiac performance.

In agreement with the results of previous studies, we did not observe any improvement in the indices of diastolic function after physical training [39], or even after MET alone or in combination with exercise. Moreover, a beneficial effect of MET on diastolic function was recently hypothesized and proposed as a rational endpoint in a randomized trial [40].

\section{Limitations}

The main limitation of the study is related to the study design. A single-blinded design was chosen due to the difficulties of concealing the exercise aspect of treatment. Moreover, patients were not randomized to a treatment group and rather were allocated a treatment based on consecutive enrollment to the study.

\section{CONCLUSION}

The primary purpose of this study was to assess the potential advantages of the association between physical training and the administration of MET on LV function in individuals with IR. Although exercise is currently recommended as a cornerstone therapy for the prevention of diabetes, adding MET has not been shown to improve cardiopulmonary performance or sensitivity to insulin.

Our results demonstrate that the combination of MET and physical training improves LV myocardial function in IR patients. Given the known positive effect on cardiopulmonary function of adding physical exercise to MET, these findings suggest the advantage of the combination of pharmacological and physical therapy to ward off the multiple adverse effects of insulin resistance on cardiovascular function.

\section{ACKNOWLEDGMENTS}

No funding or sponsorship was received for this study or publication of this article. All named authors meet the International Committee of Medical Journal Editors (ICMJE) criteria for authorship for this manuscript, take responsibility for the integrity of the work as a whole, and have given final approval to the version to be published.

Disclosures. Christian Cadeddu, Silvio Nocco, Lucia Cugusi, Martino Deidda, Orru Fabio, Stefano Bandino, Efisio Cossu, Michela Incani, Marco Giorgio Baroni and Giuseppe Mercuro have nothing to disclose.

Compliance with Ethics Guidelines. All procedures followed were in accordance with the ethical standards of the responsible committee on human experimentation (ethics committees of the University of Cagliari, Italy) and with the Helsinki Declaration of 1964, as revised in 2013. Informed consent was obtained from all patients prior to inclusion in the study.

Open Access. This article is distributed under the terms of the Creative Commons Attribution-NonCommercial 4.0 International License (http://creativecommons.org/licenses/ by-nc/4.0/), which permits any noncommercial use, distribution, and reproduction in any medium, provided you give appropriate credit to the original author(s) and the source, provide a link to the Creative Commons license, and indicate if changes were made. 


\section{REFERENCES}

1. Reaven GM, Lecture B. Role of insulin resistance in human disease. Diabetes. 1988;1988(37):1595-607.

2. Miyatake N, Saito T, Wada J, et al. Comparison of ventilatory threshold and exercise habits between Japanese men with and without metabolic syndrome. Diabetes Res Clin Pract. 2007;77(2):314-9.

3. Tuunanen H, Knuuti J. Metabolic remodelling in human heart failure. Cardiovasc Res. 2011;90:251-7.

4. Ingelsson E, Sundström J, Arnlöv J, Zethelius B, Lind $\mathrm{L}$. Insulin resistance and risk of congestive heart failure. JAMA. 2005;294:334-41.

5. Knowler WC, Barrett-Connor E, Fowler SE, et al. Reduction in the incidence of type 2 diabetes with lifestyle intervention or metformin. N Engl J Med. 2002;346:393-403.

6. Rhee MK, Herrick K, Ziemer DC, et al. Many Americans have pre-diabetes and should be considered for metformin therapy. Diabetes Care. 2010;33:49-54.

7. Nathan DM, Buse JB, Davidson MB, et al. Medical management of hyperglycemia in type 2 diabetes: a consensus algorithm for the initiation and adjustment of therapy: a consensus statement of the American Diabetes Association and the European Association for the Study of Diabetes. Diabetes Care. 2009;32:193-203.

8. Braun B, Eze P, Stephens BR, et al. Impact of metformin on peak aerobic capacity. Appl Physiol Nutr Metab. 2008;33:61-7.

9. Cadeddu C, Nocco $S$, Deidda $M$, et al. Cardiopulmonary and endothelial effects of metformin treatment in an insulin resistant population. Int J Cardiol. 2012;158:302-4.

10. Malin SK, Gerber R, Chipkin SR, Braun B. Independent and combined effects of exercise training and metformin on insulin sensitivity in individuals with prediabetes. Diabetes Care. 2012;35:131-6.

11. Cadeddu C, Nocco S, Lucia C, et al. Effects of metformin and exercise training, alone or in association, on cardio-pulmonary performance and quality of life in insulin resistance patients. Cardiovasc Diabetol. 2014;13:93.

12. Geyer H, Caracciolo G, Abe H, et al. Assessment of myocardial mechanics using speckle tracking echocardiography: fundamentals and clinical applications. J Am Soc Echocardiogr. 2010;23:351-69 (quiz 453-5).

13. Ha TH, Seo H-S, Choo WJ, et al. The effect of metabolic syndrome on myocardial contractile reserve during exercise in non-diabetic hypertensive subjects. J Cardiovasc Ultrasound. 2011;19:176-82.

14. Cadeddu C, Nocco S, Piano D, et al. Early impairment of contractility reserve in patients with insulin resistance in comparison with healthy subjects. Cardiovasc Diabetol. 2013;12:66.

15. Bonora E, Kiechl S, Willeit J, et al. Prevalence of insulin resistance in metabolic disorders: the Bruneck Study. Diabetes. 1998;47:1643-9.

16. Cadeddu C, Nocco S, Deidda M, Pau F, Colonna P, Mercuro G. Altered transmural contractility in postmenopausal women affected by cardiac syndrome X. J Am Soc Echocardiogr. 2014;27:208-14.

17. Colberg SR, Albright AL, Blissmer BJ, et al. Exercise and type 2 diabetes: American College of Sports Medicine and the American Diabetes Association: joint position statement. Exercise and type 2 diabetes. Med Sci Sports Exerc. 2010;42:2282-303.

18. Nagueh SF, Appleton CP, Gillebert TC, et al. Recommendations for the evaluation of left ventricular diastolic function by echocardiography. Eur J Echocardiogr. 2009;10:165-93.

19. Kuznetsova T, Herbots L, Richart $\mathrm{T}$, et al. Left ventricular strain and strain rate in a general population. Eur Heart J. 2008;29:2014-23.

20. de las Fuentes L, Brown AL, Mathews SJ, et al. Metabolic syndrome is associated with abnormal left ventricular diastolic function independent of left ventricular mass. Eur Heart J. 2007;28:553-9.

21. Masugata H, Senda S, Goda F, et al. Left ventricular diastolic dysfunction as assessed by echocardiography in metabolic syndrome. Hypertens Res. 2006;29:897-903.

22. Boudina S, Abel ED. Diabetic cardiomyopathy revisited. Circulation. 2007;115:3213-23.

23. Al Zadjali MA, Godfrey V, Khan F, et al. Insulin resistance is highly prevalent and is associated with reduced exercise tolerance in nondiabetic patients with heart failure. J Am Coll Cardiol. 2009;53:747-53.

24. Doehner W, Rauchhaus M, Ponikowski P, et al. Impaired insulin sensitivity as an independent risk factor for mortality in patients with stable chronic heart failure. J Am Coll Cardiol. 2005;46:1019-26. 
25. Ingelsson E, Arnlöv J, Lind L, Sundström J. Metabolic syndrome and risk for heart failure in middle-aged men. Heart. 2006;92:1409-13.

26. Witteles RM, Fowler MB. Insulin-resistant cardiomyopathy clinical evidence, mechanisms, and treatment options. J Am Coll Cardiol. 2008;51:93-102.

27. UK Prospective Diabetes Study (UKPDS) Group. Effect of intensive blood-glucose control with metformin on complications in overweight patients with type 2 diabetes (UKPDS 34. Lancet. 1998;352:854-65.

28. Bethel MA, Califf RM. Role of lifestyle and oral anti-diabetic agents to prevent type 2 diabetes mellitus and cardiovascular disease. Am J Cardiol. 2007;99:726-31.

29. Dasgupta K, Grover SA, Da Costa D, Lowensteyn I, Yale JF, Rahme E. Impact of modified glucose target and exercise interventions on vascular risk factors. Diabetes Res Clin Pract. 2006;72:53-60.

30. Cittadini A, Napoli R, Monti MG, et al. Metformin prevents the development of chronic heart failure in the SHHF rat model. Diabetes. 2012;61:944-53.

31. Wang XF, Zhang JY, Li L, Zhao XY, Tao HL, Zhang L. Metformin improves cardiac function in rats via activation of AMP-activated protein kinase. Clin Exp Pharmacol Physiol. 2011;38:94-101.

32. Masoudi FA, Inzucchi SE, Wang Y, Havranek EP, Foody JM, Krumholz HM. Thiazolidinediones, metformin, and outcomes in older patients with diabetes and heart failure: an observational study. Circulation. 2005;111:583-90.

33. Salpeter SR, Buckley NS, Kahn JA, Salpeter EE. Meta-analysis: metformin treatment in persons at risk for diabetes mellitus. Am J Med. 2008;121(149-57):e2.
34. Wong AK, Symon R, Al Zadjali MA, et al. The effect of metformin on insulin resistance and exercise parameters in patients with heart failure. Eur J Heart Fail. 2012;14:1303-10.

35. Hughes VA, Fiatarone MA, Fielding RA, et al. Exercise increases muscle GLUT-4 levels and insulin action in subjects with impaired glucose tolerance. Am J Physiol. 1993;264:E855-62.

36. Koval JA, Maezono K, Patti ME, Pendergrass M, DeFronzo RA, Mandarino LJ. Effects of exercise and insulin on insulin signaling proteins in human skeletal muscle. Med Sci Sports Exerc. 1999;31:998-1004.

37. Love-Osborne K, Sheeder J, Zeitler P. Addition of metformin to a lifestyle modification program in adolescents with insulin resistance. J Pediatr. 2008;152:817-22.

38. Snehalatha C, Mary S, Selvam S, et al. Changes in insulin secretion and insulin sensitivity in relation to the glycemic outcomes in subjects with impaired glucose tolerance in the Indian Diabetes Prevention Programme-1 (IDPP-1). Diabetes Care. 2009;32:1796-801.

39. Stewart KJ, Ouyang P, Bacher AC, Lima S, Shapiro EP. Exercise effects on cardiac size and left ventricular diastolic function: relationships to changes in fitness, fatness, blood pressure and insulin resistance. Heart. 2006;92:893-8.

40. Ladeiras-Lopes R, Fontes-Carvalho R, Bettencourt N, Sampaio F, Gama V, Leite-Moreira AF. METformin in DIastolic Dysfunction of MEtabolic syndrome (MET-DIME) trial: rationale and study design: MET-DIME trial. Cardiovasc Drugs Ther. 2014;28:191-6. 